\title{
22. MAGNETOSTRATIGRAPHY OF LOWER EOCENE TO LOWER MIOCENE SEDIMENTS IN CORES FROM THE NEW JERSEY COASTAL PLAIN ${ }^{1}$
}

\author{
Mickey C. Van Fossen²
}

\begin{abstract}
This paper describes the magnetostratigraphy of lower and middle Eocene, lower and upper Oligocene, and lower Miocene terrigenous sediments recovered in three on shore borecores on the New Jersey coastal plain (Island Beach, Atlantic City, Cape May Sites; Ocean Drilling Program Leg 150X). Near-shore, terrigenous sediments are quite often very weakly magnetized. Thus, to improve the practical measuring sensitivity of the magnetometer, I have taken high-volume $\left(120 \mathrm{~cm}^{3}\right)$, whole-round samples from these cores. Most samples were alternating-field (AF) demagnetized to $50 \mathrm{mT}$ in a large-diameter solenoid, and pilot samples from Island Beach were thermally demagnetized. At two of the three Leg 150X sites (Island Beach and Atlantic City), magnetic field polarity reversals are found in Oligocene and Eocene passive margin sequences bounded by disconformities (sequence boundaries). There are also two short, lower Miocene sections at the Cape May Site that have a series of polarity reversals. In limited stratigraphic sections, where biostratigraphic (planktonic foraminifers, calcareous nannofossils) and $\mathrm{Sr}-$ isotope data are available, correlations are made to the geomagnetic polarity time scale. These data help to date sediments at discrete stratigraphic levels in the cores and to estimate the duration of hiatuses at sequence boundaries.
\end{abstract}

\section{INTRODUCTION}

As part of the New Jersey Transect (Miller and Mountain, 1994), the overall goal of Ocean Drilling Program (ODP) onshore Leg 150X is to examine the response of sedimentation on the passive margin to glacioeustatic changes. Magnetostratigraphic studies at ODP Leg 150 Sites 903 and 904 have provided useful age data for Miocene sediments on the New Jersey upper slope (Van Fossen and Urbat, 1996). In this study, I extend the magnetostratigraphy of terrigenous sediments landward into upper shelf paleoenvironments. The integrated biostratigraphy, Sr-isotope stratigraphy, and magnetostratigraphy of Leg $150 \mathrm{X}$ helps set the stage for future offshore drilling on the middle and outer shelf (e.g., ODP Leg 174A), which will then give a more complete series of dated sections on the New Jersey passive margin (Miller and Mountain, 1994).

A difficulty facing magnetostratigraphy in the passive margin setting is that near-shore marine deposition is very often interrupted by relatively abrupt breaks in sedimentation (disconformities) brought on by changes in sea level and/or sediment influx from the continent. Consequently, one must extract magnetostratigraphic records from relatively short sections of sediments (sequences) bounded by major breaks in lithology (sequence boundaries). In addition, rapidly varying sedimentation rates will hinder attempts at the simple matching of polarity reversal patterns to the geomagnetic polarity time scale (GPTS). Thus, the key to making age assignments based on the GPTS is to perform "integrated stratigraphy" by incorporating all available methods of characterizing and dating sediments (i.e., physical stratigraphy, biostratigraphy, and Sr-isotope stratigraphy). The Berggren et al. (1995) GPTS is used in this study, and, where identified, reversal boundaries are referenced to onsets or terminations of magnetochrons using the suffixes "O" or "T," respectively.

The intensity of remanent magnetism in terrigenous sediments is often very weak owing to the high energy of deposition of the paleoenvironment. However, recent magnetostratigraphic studies have im-

${ }^{1}$ Miller, K.G., and Snyder, S.W. (Eds.), 1997. Proc. ODP, Sci. Results, 150X: College Station, TX (Ocean Drilling Program).

${ }^{2}$ Department of Geological Sciences, Rutgers University, Piscataway, NJ 08855 , U.S.A. (Present address: bsquare inc., 3633 136th Place SE, Suite 100, Bellevue, WA 98006, U.S.A.) mickvf@ bsquare.com proved on the success rate in this environment by simply increasing the volume of material per sample, and thereby increasing the magnetic signal-to-noise ratio (Miller et al., 1990, 1993; Van Fossen and Urbat, 1996). Another strategy in these studies has been to sample the passive margin by way of continental or deep-sea drilling, which eliminates problems apparently related to weathering in outcrops (Ellwood et al., 1986). In this study, these methods are extended to three onshore sites of Leg 150X (Island Beach, Atlantic City, and Cape May). By way of large-volume sampling, I have been able to recover magnetostratigraphies from weakly magnetized lower and middle Eocene, lower and upper Oligocene, and lower Miocene terrigenous sediments.

\section{MAGNETOSTRATIGRAPHY}

Paleomagnetic samples from cores of Leg $150 \mathrm{X}$ are mainly whole-round samples $(4.32-\mathrm{cm}$ diameter) with a nominal volume of $120 \mathrm{~cm}^{3}$, i.e., twenty times that of the conventional ODP paleomagnetic sample. This amounts to a twenty-fold increase in signal-tonoise ratio on the cryogenic magnetometer, and I estimate that such an increase improves the practical sensitivity of magnetization intensity to better than $0.01 \mathrm{~mA} / \mathrm{m}$. Similarly, at Sites 903 and 904 of Leg 150 , quarter-core samples $42 \mathrm{~cm}^{3}$ in volume were used to improve the measurement sensitivity level (Van Fossen and Urbat, 1996). The data from those samples formed the basis of the magnetostratigraphy, replacing virtually all of the shipboard pass-through data, which was marred by a core liner magnetization and spurious magnetizations attributed to drilling slurry. Terrigenous sediments are often coarsely grained and the detrital and chemical sources of the iron-oxide grains can be mixed. Thus depositional processes in the near-shore paleoenvironment can result in a very "inefficient" remanent magnetization. A large-volume sample can also help overcome this problem by providing a more representative sample of the magnetization in the sediment. The magnetizations in the terrigenous sediments of Leg 150X were in general very weak, and the success of measurement indeed depended on a greater sample size. There were, however, limited sections at the Island Beach (IB) and Cape May (CM) sites where intensities were strong enough to be sampled with the conventional $6-\mathrm{cm}^{3}$ sample. 
The paleomagnetism laboratory at the Lamont-Doherty Earth Observatory (LDEO) is equipped with a large-volume alternating-field (AF) demagnetization coil in which samples up to $\sim 300 \mathrm{~cm}^{3}$ can be demagnetized to $50 \mathrm{mT}$. Two large-access ovens with internal fields $<10 \mathrm{nT}$ are also available for thermal demagnetization. These apparatus enable the decomposition of the natural remanent magnetization (NRM) to reveal the most stable component of magnetization in individual samples. All measurements of magnetic remanence were made on a $2 \mathrm{G}$ cryogenic three-axis magnetometer. Measurement and demagnetization experiments were conducted within a shielded room with a nominal field of $\sim 200 \mathrm{nT}$. In each sample, the most stable magnetization vector was identified through analysis of demagnetization data in orthographic projection. The method of least-squares analysis (Kirschvink, 1980) was applied to the demagnetization trajectories to calculate least-squares best-fitting vectors, with inclination forming the basis of polarity determination. An estimate of the percentage of total variance in the selected data and an intensity of the best-fit magnetization vector are also provided by the least-squares analysis. These parameters are used in evaluating the data.

\section{Island Beach Site (Site IB)}

Site IB is located at $39^{\circ} 48^{\prime} \mathrm{N}, 74^{\circ} 05^{\prime} \mathrm{W}$ in Island Beach State Park, New Jersey, at a mean elevation of $3.7 \mathrm{~m}$ (Fig. 1). Drilling to $1223 \mathrm{ft}$ $(372.8 \mathrm{~m})$ recovered $1060 \mathrm{ft}(323.1 \mathrm{~m})$ of core. The lower Miocene Kirkwood Formation could not be sampled, because it was generally unconsolidated at Site IB, and drilling with the Christensen smalldiameter system did not begin until $453 \mathrm{ft}$ (138.1 m; Miller et al., 1994b). Paleomagnetic work begins in the upper Oligocene Atlantic City Formation (505-693 ft [153.9-211.2 m]) where the sediments are more lithified and suitable for sampling. At Site IB, a portion of the sample collection was subjected to thermal demagnetization as a check on AF treatments. The sample chosen for thermal demagnetization in most cases has a nearby AF-treated sample (usually within $2 \mathrm{ft}[0.6 \mathrm{~m}])$ for a better comparison of the two methods. In all cases, thermal demagnetization confirmed the $\mathrm{AF}$ results, although treatments beyond about $500^{\circ} \mathrm{C}$ induced spurious magnetizations in the samples, probably the result of magnetochemical alteration during heating.

Magnetization intensities are fairly strong $(1-10 \mathrm{~mA} / \mathrm{m})$ in the dark green, glauconitic clayey sands of the lower Oligocene (Sewell Point Formation, 602-697 ft [183.5-212.4 m]), but reduce to 0.01 $\mathrm{mA} / \mathrm{m}$ at the base of the section, where the sediments become dark gray to black and more lignite-rich (656-697 ft [199.9-212.4 m]). AF-demagnetization experiments suggest a single component of magnetization throughout the Oligocene section (Fig. 2), and only three samples were rejected owing to non-interpretable demagnetization profiles. The data suggest three polarity zones in the Oligocene section between depths of 523.1 and $658.5 \mathrm{ft}$ (159.4 and $200.7 \mathrm{~m}$ ).

The lowermost Oligocene (Sewell Point Formation) and middle to upper Eocene (Absecon Inlet Formation) at Site IB were sampled from 693.5 to $789.9 \mathrm{ft}(211.4-240.7 \mathrm{~m})$. These glauconitic clays, silty clays, and clayey sands are generally weakly magnetized $(\sim 0.01$ $\mathrm{mA} / \mathrm{m}$; Table 1). A number of samples were either too weak to measure or gave inconsistent directions of magnetization during progressive demagnetization; however, the data from seven samples were salvaged and indicate a relatively thick zone of reversely magnetized sediments (Table 1). In the upper part of this zone, the inclination of remanence is relatively shallow, usually less than $25^{\circ}$. The primary reversed component of magnetization may be contaminated by a normal polarity overprint that is often incompletely removed from the total magnetization in samples from this section.

Demagnetization of sandy glauconite clay from the middle Eocene Shark River Formation (800-862 ft [243.8-262.7 m]) yields a relatively weak $(0.01 \mathrm{~mA} / \mathrm{m})$, but very consistent magnetization. I eliminated data from the four samples that were taken in the upper, more glauconitic part of the Shark River Formation $(800-825 \mathrm{ft}$

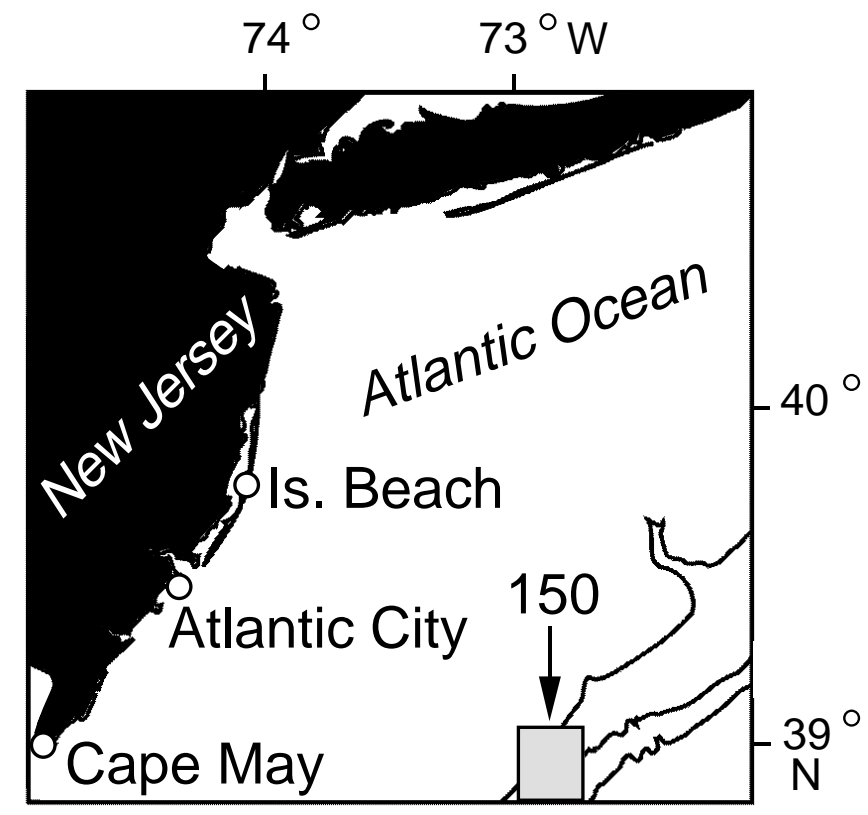

Figure 1. Location map of the New Jersey Coastal Plain and Leg 150X sites at Island Beach, Atlantic City, and Cape May. Square indicates ODP Leg 150 study area.

[243.8-251.5 m]) where the direction of magnetization behaved very erratically during laboratory treatment. Below a disconformity at 862 $\mathrm{ft}(262.7 \mathrm{~m})$, greenish gray clay and sandy clay of the lower Eocene Manasquan Formation are also relatively weakly magnetized, yet yield very consistent and straightforward demagnetization profiles (Fig. 3; Table 1). Sample B870.0 demonstrates thermal demagnetization of a fine sandy clay (Fig. 3). In the Manasquan Formation and Shark River Formation above, zones of reversed and normal polarity magnetization are present throughout, and there is little evidence for contamination by any secondary magnetization.

Below the contact at $1076 \mathrm{ft}(328.0 \mathrm{~m})$, sampling was possible only in scattered sections where the core material was not disturbed by drilling. Samples from upper Paleocene sediments $(1076-1167 \mathrm{ft}$ [328.0-355.7 m]) are mainly normal in polarity, but two samples at $1119.0 \mathrm{ft}(341.1 \mathrm{~m})$ and $1128.6 \mathrm{ft}(344.0 \mathrm{~m})$ are reversely magnetized (Table 1). I have also measured a reversed polarity magnetization in two samples from the upper Cretaceous Navesink Formation (1197.6 and $1219.5 \mathrm{ft}$ [ 365.0 and $371.7 \mathrm{~m}$ ]). Although it was not possible to develop a magnetostratigraphy in these sediments, the data suggest that these pre-Eocene New Jersey Coastal Plain formations have the potential to record an ancient magnetization.

In summary, at Site IB I have progressively AF demagnetized and analyzed 118 whole-round and standard 6- $\mathrm{cm}^{3}$ samples, 29 of which were omitted from magnetostratigraphic analysis owing to a weak and/or non-interpretable magnetization. Based on the data from the remaining 89 samples, I incorporated the biostratigraphic and Srisotope data from Site IB to make correlations to the GPTS.

A polarity transition occurs at $549.95 \mathrm{ft}(167.6 \mathrm{~m})$, roughly in the middle of the upper Oligocene Atlantic City Formation at Site IB (Fig. 2). In their reexamination of the planktonic foraminifers, Pekar et al. (Chapter 15, this volume) judge this section as reworked and note the Sr-isotope date of $24.3 \mathrm{Ma}$ at $520 \mathrm{ft}$ (158.5 m; Sugarman et al., Chapter 12 , this volume). Thus the previous age assignment of these sediments to Zone P20-P21a (Miller et al., 1994b) is probably too old. In addition, Pekar et al. (Chapter 15, this volume) have made a cross correlation to Zone P22 sediments at Site CM by way of gamma-ray logs, suggesting the Atlantic City Formation at Island Beach is also latest Oligocene in age. Unfortunately, there is no subdivision of Zone P22 


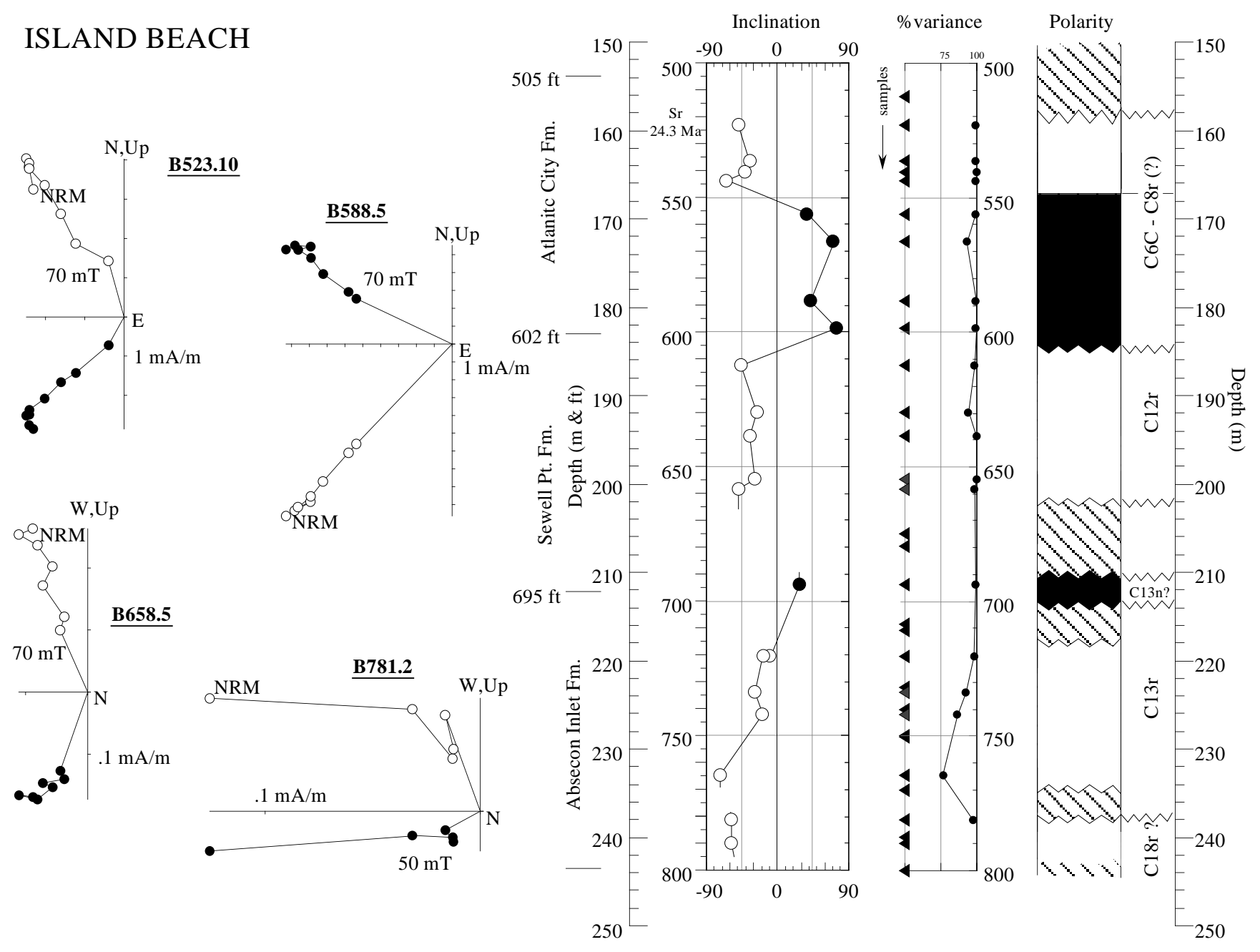

Figure 2. Progressive AF demagnetization and magnetostratigraphy of samples from Site IB (500-800 ft [152.4-243.8 m]). Demagnetization profiles in orthographic projection are shown at left (closed/open symbols = horizontal/vertical projection, $\mathrm{mT}=$ millitesla). Inclination of remanence vs. depth shown on right along with percent variance (triangles give location of discrete samples). Polarity interpretation (black $=$ normal, white $=$ reverse, hatch $=$ uninterpretable).

or available nannofossil data from this section. Also, the simple polarity zonation of reverse $(523.10-549.95 \mathrm{ft}$ [159.4-167.6 m]) and normal (549.95-598.30 ft [167.6-182.4 m]) is insufficient to venture a precise correlation to the GPTS. These polarity zones can correlate to any late Oligocene chron from C6C to C8r.

Below the disconformity at $602 \mathrm{ft}(183.5 \mathrm{~m})$, the lower Oligocene Sewell Point Formation has been assigned to planktonic foraminifer Zone P19 (Miller et al, 1994b, Pekar et al., Chapter 15, this volume). Therefore, the thick zone of reversed polarity between 612 and $659 \mathrm{ft}$ (186.5 and $200.8 \mathrm{~m}$ ) at Site IB is likely to correlate to Chron C12r, a relatively lengthy period of reversed magnetic field polarity ( 2 m.y.) in the early Oligocene, according to the GPTS. Sr-isotope dates from this section range from 32.7 to $33.1 \mathrm{Ma}$ (Sugarman et al., Chapter 12, this volume) and agree with an assignment to Chron C12r. Thus, given the Oligocene chronology at Site IB, it is suggested that the disconformity at $602 \mathrm{ft}(183.5 \mathrm{~m})$ between the Atlantic City and Sewell Point Formations represents a hiatus of roughly 6 m.y.

A precise planktonic foraminiferal zonation is not available for the upper Eocene Absecon Inlet Formation due to the absence of Cribrohantkenina inflata (Miller et al., 1994b). However, M.-P. Aubry (pers. comm., 1995) has assigned the glauconitic silty clay section between 710 and $752 \mathrm{ft}(216.4$ and $229.2 \mathrm{~m})$ to calcareous nannoplankton Zone NP19/20. It is therefore suggested that the re- versed polarity zone between 720.5 and $764.5 \mathrm{ft}(219.6$ and $233.0 \mathrm{~m})$ correlates to the older half of Chron C13r (although a correlation to $\mathrm{C} 15 \mathrm{r}$ is also possible).

Between the depths of 800 and $900 \mathrm{ft}$ (243.8 and $274.3 \mathrm{~m}$ ), sediments within the lower Shark River Formation (middle Eocene) and upper Manasquan Formation (lower Eocene) were deposited in a rapid series of five passive margin sequences (E4 through E8, Browning et al., Chapter 16, this volume). These sequences are identified primarily through lithostratigraphy and benthic foraminiferal biofacies. The identification of five polarity intervals between 835 and $930 \mathrm{ft}$ (254.5 and $283.5 \mathrm{~m}$ ) and nannofossil data (M.-P.Aubry, pers. comm., 1995) help in determining precise ages of sediments and durations of hiatuses represented by the sequence boundaries (see Table 2; Fig. 3). Nannofossils at $840 \mathrm{ft}(256.0 \mathrm{~m})$ indicate Zone NP15c (M.-P. Aubry, pers. comm., 1995), and therefore the normal polarity interval above sequence Boundary E5/6 (846 ft [257.9 m]) is probably Chron C20n. Below $851 \mathrm{ft}(259.4 \mathrm{~m})$, down to the disconformity at $857 \mathrm{ft}$ (261.2 m; Miller et al., 1994a; M.-P.Aubry, pers. comm., 1995), three samples define Chron C21n partim (Table 2; Fig. 3). Based on the GPTS, an age of $46.3 \mathrm{Ma}$ is assigned to the sediments at a depth of $851.00 \mathrm{ft}$ (C21n termination) and 49.6 Ma to the sediments at 867.75 $\mathrm{ft}$ (264.5 m; C22n onset; see Table 2). Chron 21r is missing from this section; thus, the sequence boundaries at 857 and $862 \mathrm{ft}$ (261.2 and 
Table 1. Progressive demagnetization data used in constructing Site IB (Island Beach) magnetostratigraphy.

\begin{tabular}{|c|c|c|c|c|c|c|c|c|}
\hline $\begin{array}{c}\text { Sample } \\
\text { name }\end{array}$ & $\begin{array}{l}\text { Depth } \\
\text { (m) }\end{array}$ & $\mathrm{N}$ & $\begin{array}{l}\text { Var } \\
(\%)\end{array}$ & $\begin{array}{c}\text { Dec } \\
\left({ }^{\circ}\right)\end{array}$ & $\begin{array}{l}\text { Inc } \\
\left({ }^{\circ}\right)\end{array}$ & $\begin{array}{l}\text { First } \\
(\mathrm{mT})\end{array}$ & $\begin{array}{l}\text { Last } \\
(\mathrm{mT})\end{array}$ & $\begin{array}{c}\text { Jcomp } \\
(\mathrm{mA} / \mathrm{m})\end{array}$ \\
\hline \multicolumn{9}{|l|}{ 150X-IB- } \\
\hline B523.10 & 159.44 & 5 & 99.7 & 223.8 & -48.7 & 20.0 & 70.0 & 5.0783 \\
\hline B536.50 & 163.53 & 5 & 98.9 & 105.4 & -34.2 & 20.0 & 70.0 & 2.5750 \\
\hline B540.30 & 164.68 & 7 & 99.9 & 305.1 & -41.4 & 100.0 & 400.0 & 9.9070 \\
\hline B543.60 & 165.69 & 7 & 99.4 & 317.7 & -64.8 & 20.0 & 80.0 & 3.8917 \\
\hline B556.30 & 169.56 & 5 & 99.4 & 216.5 & 37.0 & 20.0 & 70.0 & 3.2417 \\
\hline B566.20 & 172.58 & 5 & 93.0 & 198.9 & 69.6 & 20.0 & 70.0 & 1.8767 \\
\hline B588.50 & 179.37 & 4 & 99.7 & 310.8 & 41.0 & 20.0 & 50.0 & 4.5200 \\
\hline B598.30 & 182.36 & 4 & 99.2 & 291.3 & 74.2 & 20.0 & 50.0 & 6.5067 \\
\hline B612.15 & 186.58 & 7 & 98.7 & 95.2 & -45.6 & 20.0 & 80.0 & 14.5383 \\
\hline B629.90 & 191.99 & 7 & 93.8 & 211.7 & -25.0 & 100.0 & 400.0 & 1.9017 \\
\hline B638.30 & 194.55 & 7 & 99.9 & 159.9 & -34.3 & 20.0 & 80.0 & 5.3083 \\
\hline B654.80 & 199.58 & 6 & 99.8 & 316.6 & -29.0 & 5.0 & 70.0 & 0.2321 \\
\hline B658.50 & 200.71 & 6 & 98.8 & 115.1 & -48.7 & 5.0 & 70.0 & 0.0268 \\
\hline B693.50 & 211.38 & 4 & 99.1 & 133.3 & 27.9 & 35.0 & 50.0 & 0.0158 \\
\hline B720.50 & 219.61 & 3 & 98.3 & 98.4 & -10.0 & 30.0 & 50.0 & 0.0263 \\
\hline B733.60 & 223.60 & 6 & 91.9 & 110.3 & -28.3 & 7.5 & 40.0 & 0.0435 \\
\hline B742.00 & 226.16 & 5 & 86.6 & 230.2 & -18.8 & 30.0 & 50.0 & 0.0368 \\
\hline B764.50 & 233.02 & 6 & 76.4 & 329.9 & -72.4 & 20.0 & 50.0 & 0.0404 \\
\hline B781.20 & 238.11 & 4 & 97.9 & 150.6 & -59.2 & 20.0 & 50.0 & 0.0576 \\
\hline B789.90 & 240.76 & 1 & - & 165.0 & -58.6 & 40.0 & 40.0 & 0.0103 \\
\hline B840.50 & 256.18 & 5 & 93.0 & 177.7 & 62.1 & 10.0 & 50.0 & 0.0164 \\
\hline B844.40 & 257.37 & 4 & 98.8 & 145.3 & 48.7 & 20.0 & 50.0 & 0.0303 \\
\hline B844.80 & 257.50 & 6 & 93.6 & 156.2 & 22.2 & 5.0 & 50.0 & 0.0321 \\
\hline B848.00 & 258.47 & 5 & 97.1 & 197.4 & -10.6 & 5.0 & 40.0 & 0.0159 \\
\hline B850.00 & 259.08 & 7 & 84.8 & 117.4 & -57.5 & 200.0 & 480.0 & 0.0852 \\
\hline B852.00 & 259.69 & 6 & 84.1 & 247.5 & 32.9 & 5.0 & 40.0 & 0.0097 \\
\hline B855.00 & 260.60 & 5 & 99.4 & 97.6 & 34.4 & 5.0 & 40.0 & 0.0238 \\
\hline B857.15 & 261.26 & 5 & 98.1 & 137.7 & 52.6 & 5.0 & 40.0 & 0.0208 \\
\hline B859.85 & 262.08 & 5 & 96.2 & 243.4 & 38.6 & 5.0 & 40.0 & 0.0159 \\
\hline B864.30 & 263.44 & 4 & 99.3 & 213.8 & 50.8 & 20.0 & 50.0 & 1.1191 \\
\hline B866.30 & 264.05 & 5 & 98.3 & 292.8 & 52.9 & 5.0 & 40.0 & 0.0355 \\
\hline B869.20 & 264.93 & 4 & 97.7 & 217.7 & -40.0 & 20.0 & 50.0 & 0.0562 \\
\hline B870.00 & 265.18 & 7 & 65.5 & 18.2 & -52.0 & 200.0 & 480.0 & 0.0071 \\
\hline B871.30 & 265.57 & 4 & 81.8 & 267.4 & 8.6 & 30.0 & 50.0 & 0.0052 \\
\hline B871.90 & 265.76 & 6 & 99.8 & 276.3 & 16.9 & 15.0 & 50.0 & 1.6674 \\
\hline B873.45 & 266.23 & 6 & 97.8 & 238.6 & -1.3 & 10.0 & 50.0 & 0.0162 \\
\hline B875.40 & 266.82 & 4 & 99.8 & 69.3 & -18.5 & 30.0 & 50.0 & 0.0095 \\
\hline B876.75 & 267.23 & 5 & 96.3 & 177.1 & 55.7 & 7.5 & 50.0 & 0.0317 \\
\hline B885.30 & 269.84 & 5 & 98.0 & 77.6 & 10.8 & 7.5 & 50.0 & 0.0179 \\
\hline B888.25 & 270.74 & 5 & 96.0 & 160.8 & 12.2 & 7.5 & 50.0 & 0.0273 \\
\hline B889.50 & 271.12 & 6 & 62.6 & 228.7 & 18.8 & 250.0 & 480.0 & 0.0037 \\
\hline B907.00 & 276.45 & 5 & 96.6 & 215.6 & 25.1 & 10.0 & 50.0 & 0.0230 \\
\hline B922.60 & 281.21 & 4 & 88.3 & 282.6 & 54.0 & 20.0 & 50.0 & 0.0197 \\
\hline B934.30 & 284.77 & 1 & - & 43.3 & -54.4 & 50.0 & 50.0 & 0.0142 \\
\hline B940.80 & 286.76 & 4 & 86.0 & 234.3 & 9.1 & 20.0 & 50.0 & 0.0554 \\
\hline B944.10 & 287.76 & 3 & 91.5 & 270.5 & -58.0 & 40.0 & 50.0 & 0.0113 \\
\hline B947.20 & 288.71 & 3 & 99.4 & 192.8 & 68.7 & 30.0 & 50.0 & 0.0570 \\
\hline B955.50 & 291.24 & 4 & 90.3 & 214.6 & -31.9 & 20.0 & 50.0 & 0.0323 \\
\hline B963.00 & 293.52 & 7 & 99.2 & 347.7 & -37.9 & 300.0 & 480.0 & 0.6910 \\
\hline B969.50 & 295.50 & 4 & 94.8 & 218.7 & -12.8 & 20.0 & 50.0 & 0.0177 \\
\hline B972.00 & 296.27 & 5 & 95.8 & 187.6 & -53.0 & 10.0 & 50.0 & 0.0353 \\
\hline B974.00 & 296.88 & 4 & 94.7 & 68.9 & 62.1 & 20.0 & 50.0 & 0.0145 \\
\hline B976.00 & 297.48 & 5 & 79.6 & 120.1 & 38.2 & 10.0 & 50.0 & 0.0188 \\
\hline B978.60 & 298.28 & 4 & 97.9 & 335.6 & 74.4 & 20.0 & 50.0 & 0.0164 \\
\hline B982.40 & 299.44 & 4 & 97.3 & 211.4 & 21.2 & 20.0 & 50.0 & 0.0235 \\
\hline B982.90 & 299.59 & 5 & 94.7 & 336.4 & -48.4 & 330.0 & 450.0 & 0.0032 \\
\hline B983.40 & 299.74 & 6 & 95.9 & 337.4 & -18.0 & 30.0 & 50.0 & 0.0093 \\
\hline B984.60 & 300.11 & 5 & 88.8 & 315.0 & 45.3 & 10.0 & 50.0 & 0.0455 \\
\hline B986.60 & 300.72 & 5 & 74.2 & 67.3 & 69.1 & 10.0 & 50.0 & 0.0203 \\
\hline B991.00 & 302.06 & 4 & 95.6 & 228.9 & 77.2 & 20.0 & 50.0 & 0.0492 \\
\hline B994.75 & 303.20 & 5 & 94.3 & 332.2 & 63.9 & 20.0 & 50.0 & 0.0253 \\
\hline B1000.80 & 305.04 & 6 & 62.1 & 163.9 & 34.5 & 300.0 & 450.0 & 0.0160 \\
\hline B1006.25 & 306.70 & 4 & 99.1 & 143.6 & 29.7 & 10.0 & 50.0 & 0.0521 \\
\hline B1010.30 & 307.94 & 4 & 83.5 & 356.0 & 73.8 & 20.0 & 50.0 & 0.0126 \\
\hline B1012.50 & 308.61 & 4 & 98.7 & 124.1 & 39.4 & 20.0 & 50.0 & 0.0268 \\
\hline B1017.60 & 310.16 & 4 & 99.4 & 205.7 & 28.0 & 10.0 & 50.0 & 0.0403 \\
\hline
\end{tabular}

$262.7 \mathrm{~m}$ ) form a concatenated normal interval of $\mathrm{C} 21 \mathrm{n}$ partim and C22n partim (Fig. 3). Based on the GPTS, these breaks in sedimentation represent a cumulative hiatus of at least 1 m.y., but no greater than 3 m.y. M.-P. Aubry (pers. comm., 1995) has also identified Zone NP12 (879-955 ft [267.9-291.1 m]), and therefore I identify the thick normal polarity interval down to $930 \mathrm{ft}(283.5 \mathrm{~m})$ as Chron $\mathrm{C} 23 \mathrm{n}$. The top of $\mathrm{C} 23 \mathrm{n}$ is unfortunately truncated by sequence Boundary E3/E4 at $876 \mathrm{ft}(267.0 \mathrm{~m})$.

Following a $20-\mathrm{ft}(6.1 \mathrm{~m})$ zone of uncertain polarity zonation, the series of early Eocene magnetochrons continues with Chron $\mathrm{C} 23 \mathrm{r}$ (partim) from 955 to $973.0 \mathrm{ft}$ (291.1-296.6 m; Fig. 3). I have made this assignment based on planktonic foraminifers that suggest the sediments above $975 \mathrm{ft}(297.2 \mathrm{~m})$ are no older than Zone P7 (Miller et al., 1994b) and the calcareous nannofossils (M.-P.Aubry, pers. comm., 1995), which extend Zone NP12 down to a depth of $955 \mathrm{ft}$ (291.1 m). I also identify Chron C24n between 973.0 and $1018.8 \mathrm{ft}$ (296.6 and $310.5 \mathrm{~m}$; Fig. 3), based on the nannofossil evidence that indicates that Zone NP10 does not begin until the depth of $1022 \mathrm{ft}$ $(311.5 \mathrm{~m})$. The thin reversed polarity zone $(982.65-984.00 \mathrm{ft}$ [299.5$299.9 \mathrm{~m}]$ ) is assigned to a composite of Subchrons C24n.1r and C24n.2 (52.54-52.79 Ma; see Table 2).

\section{Atlantic City Site (Site AC)}

The Atlantic City Site (Site AC) is located at $39^{\circ} 22^{\prime} \mathrm{N}, 74^{\circ} 25^{\prime} \mathrm{W}$ in the Atlantic City Coast Guard Station at a mean elevation of $1.5 \mathrm{~m}$ (Fig. 1). Drilling to $1452 \mathrm{ft}(442.6 \mathrm{~m})$ recovered $977 \mathrm{ft}(297.8 \mathrm{~m})$ of core, and paleomagnetic sampling began at $808 \mathrm{ft}$ (246.3 m). Howev- 
Table 1 (continued).

\begin{tabular}{ccccrrrrr}
\hline $\begin{array}{c}\text { Sample } \\
\text { name }\end{array}$ & $\begin{array}{c}\text { Depth } \\
(\mathrm{m})\end{array}$ & $\mathrm{N}$ & $\begin{array}{c}\text { Var } \\
(\%)\end{array}$ & $\begin{array}{c}\text { Dec } \\
\left({ }^{\circ}\right)\end{array}$ & $\begin{array}{r}\text { Inc } \\
\left({ }^{\circ}\right)\end{array}$ & $\begin{array}{r}\text { First } \\
(\mathrm{mT})\end{array}$ & $\begin{array}{r}\text { Last } \\
(\mathrm{mT})\end{array}$ & $\begin{array}{c}\text { Jcomp } \\
(\mathrm{mA} / \mathrm{m})\end{array}$ \\
\hline B1020.40 & 311.02 & 3 & 99.3 & 288.6 & -42.9 & 30.0 & 50.0 & 0.0290 \\
B1024.65 & 312.31 & 4 & 99.6 & 304.4 & -45.4 & 20.0 & 50.0 & 0.1633 \\
B1027.00 & 313.03 & 6 & 95.0 & 172.4 & -18.6 & 20.0 & 50.0 & 0.0437 \\
B1028.80 & 313.58 & 4 & 95.8 & 108.4 & -28.5 & 20.0 & 50.0 & 0.0406 \\
B1032.60 & 314.74 & 4 & 68.7 & 296.1 & 76.6 & 20.0 & 50.0 & 0.0278 \\
B1034.90 & 315.44 & 4 & 87.5 & 184.7 & -58.8 & 20.0 & 50.0 & 0.0408 \\
B1038.40 & 316.50 & 4 & 85.4 & 191.7 & 42.8 & 20.0 & 50.0 & 0.0284 \\
B1042.50 & 317.75 & 5 & 92.0 & 299.3 & -50.0 & 200.0 & 360.0 & 0.0075 \\
B1043.20 & 317.97 & 4 & 96.0 & 160.7 & 32.2 & 20.0 & 50.0 & 0.0469 \\
B1062.90 & 323.97 & 6 & 97.1 & 25.6 & 12.0 & 10.0 & 50.0 & 0.0244 \\
B1069.50 & 325.98 & 4 & 99.4 & 221.1 & -66.2 & 20.0 & 50.0 & 0.0567 \\
B1090.50 & 332.38 & 4 & 98.2 & 146.3 & 51.8 & 20.0 & 50.0 & 0.0616 \\
B1110.50 & 338.48 & 4 & 95.4 & 174.5 & 61.8 & 20.0 & 50.0 & 0.0443 \\
B1119.00 & 341.07 & 4 & 93.3 & 192.2 & -11.1 & 250.0 & 360.0 & 0.0537 \\
B1128.60 & 344.00 & 4 & 93.4 & 223.3 & -38.1 & 20.0 & 50.0 & 0.0245 \\
B1132.40 & 345.16 & 5 & 83.7 & 194.7 & 24.9 & 10.0 & 50.0 & 0.0408 \\
B1148.90 & 350.18 & 5 & 95.5 & 180.8 & 63.3 & 10.0 & 50.0 & 0.0581 \\
B1150.30 & 350.61 & 4 & 88.9 & 259.1 & 36.5 & 20.0 & 50.0 & 0.0303 \\
B1158.50 & 353.11 & 6 & 93.6 & 227.6 & 21.3 & 200.0 & 390.0 & 0.0421 \\
B1170.20 & 356.68 & 4 & 90.7 & 223.6 & 60.7 & 20.0 & 50.0 & 0.1338 \\
B1188.70 & 362.32 & 4 & 99.0 & 37.3 & 25.9 & 20.0 & 50.0 & 0.0698 \\
B1197.60 & 365.03 & 7 & 95.7 & 110.0 & -45.9 & 150.0 & 390.0 & 0.1239 \\
B1219.50 & 371.70 & 6 & 96.9 & 322.4 & -61.1 & 150.0 & 360.0 & 0.0491
\end{tabular}

Note: $\mathrm{N}=$ number of data used in each least-squares analysis; Var = percentage of the total variance in the selected data accounted for by the least-squares vector (dash indicates variance calculation not applicable); Dec, Inc = declination and inclination of the magnetization vector; First, Last = first and last demagnetization step in millitesla; Jcomp = intensity of least-squares magnetization.

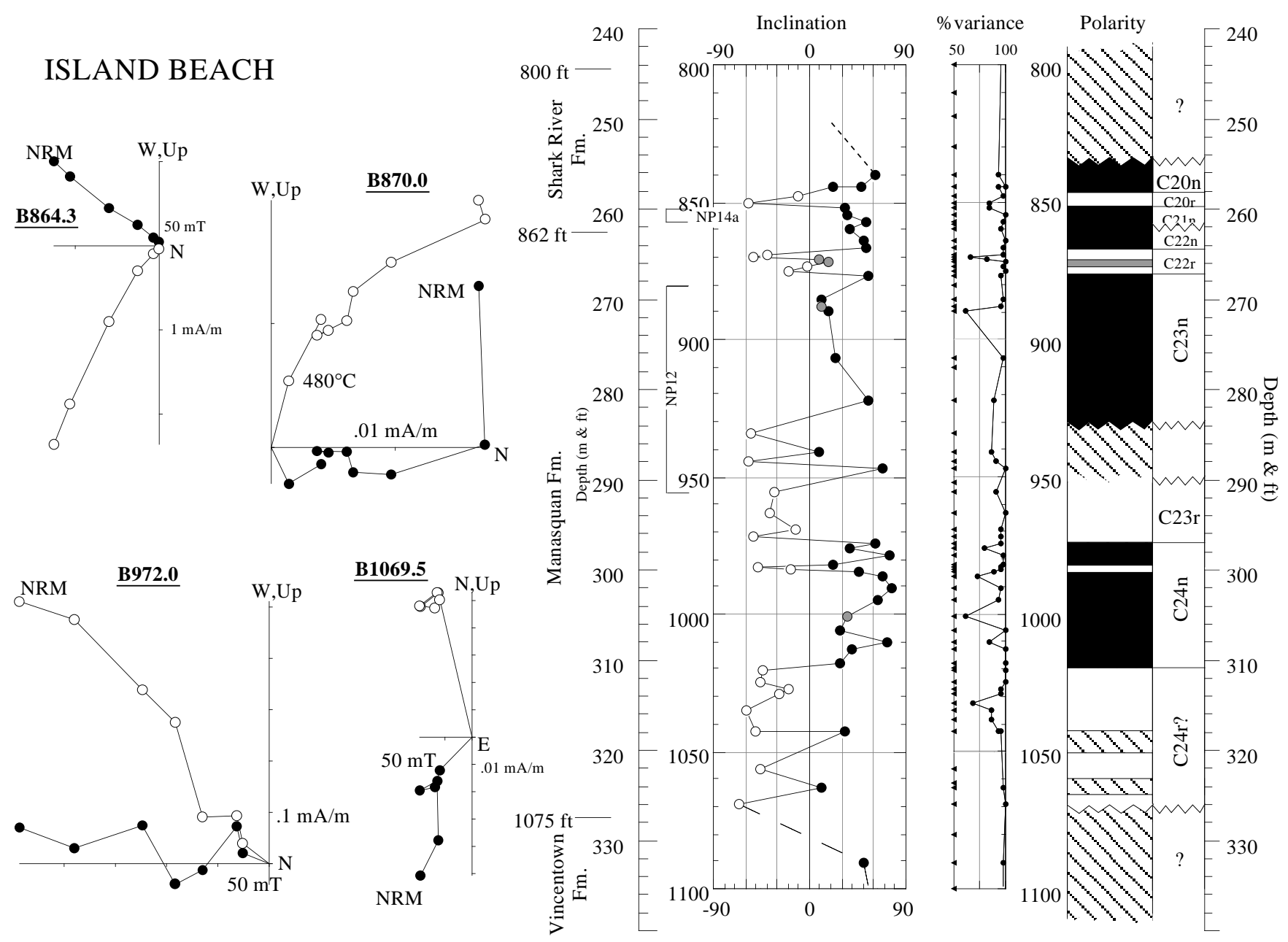

Figure 3. Progressive AF demagnetization and magnetostratigraphy of samples from Site IB (800-1100 ft [243.8-335.3 m]). Demagnetization profiles in orthographic projection are shown at left (closed/open symbols $=$ horizontal/vertical projection, $\mathrm{mT}=$ millitesla). Inclination of remanence vs. depth shown on right along with percent variance (triangles give location of discrete samples). Polarity interpretation (black $=$ normal, white $=$ reverse, hatch $=$ uninterpretable). 
Table 2. Progressive demagnetization data used in constructing Site AC (Atlantic City) magnetostratigraphy.

\begin{tabular}{|c|c|c|c|c|c|c|c|c|}
\hline $\begin{array}{c}\text { Sample } \\
\text { name }\end{array}$ & $\begin{array}{l}\text { Depth } \\
\text { (m) }\end{array}$ & $\mathrm{N}$ & $\begin{array}{l}\text { Var } \\
(\%)\end{array}$ & $\begin{array}{l}\text { Dec } \\
\left({ }^{\circ}\right)\end{array}$ & $\begin{array}{l}\text { Inc } \\
(\%)\end{array}$ & $\begin{array}{l}\text { First } \\
(\mathrm{mT})\end{array}$ & $\begin{array}{l}\text { Last } \\
(\mathrm{mT})\end{array}$ & $\begin{array}{c}\text { Jcomp } \\
(\mathrm{mA} / \mathrm{m})\end{array}$ \\
\hline \multicolumn{9}{|l|}{$150 \mathrm{X}-\mathrm{AC}-$} \\
\hline A808.50 & 246.43 & 4 & 99.1 & 299.8 & -62.5 & 20.0 & 50.0 & 0.1054 \\
\hline A919.00 & 280.11 & 4 & 99.9 & 122.8 & -42.1 & 20.0 & 50.0 & 1.9957 \\
\hline A 1000.50 & 304.95 & 4 & 99.9 & 203.3 & 51.3 & 20.0 & 50.0 & 7.1105 \\
\hline A1054.60 & 321.44 & 4 & 99.9 & 216.8 & 55.0 & 20.0 & 50.0 & 0.6413 \\
\hline A1079.40 & 329.00 & 4 & 100.0 & 217.3 & -19.8 & 20.0 & 50.0 & 1.4224 \\
\hline A1148.30 & 350.00 & 3 & 93.4 & 212.3 & 57.0 & 30.0 & 50.0 & 0.0608 \\
\hline A1177.00 & 358.75 & 3 & 87.5 & 55.6 & -14.1 & 30.0 & 50.0 & 0.0106 \\
\hline A1202.40 & 366.49 & 5 & 97.4 & 160.8 & 30.5 & 10.0 & 50.0 & 0.1132 \\
\hline A1207.00 & 367.89 & 2 & 96.8 & 115.9 & 31.3 & 40.0 & 50.0 & 0.0567 \\
\hline A1212.50 & 369.57 & 6 & 97.4 & 147.6 & 43.4 & 10.0 & 50.0 & 0.0814 \\
\hline A1216.45 & 370.77 & 6 & 95.7 & 146.8 & 36.9 & 10.0 & 50.0 & 0.0797 \\
\hline A1221.60 & 372.34 & 5 & 98.5 & 208.4 & 55.5 & 10.0 & 50.0 & 0.0641 \\
\hline A1225.20 & 373.44 & 6 & 97.4 & 85.2 & 20.2 & 10.0 & 50.0 & 0.0836 \\
\hline A1236.50 & 376.89 & 6 & 97.0 & 193.7 & 29.4 & 10.0 & 50.0 & 0.0851 \\
\hline A1239.00 & 377.65 & 4 & 95.2 & 273.6 & 65.2 & 20.0 & 50.0 & 0.0247 \\
\hline A 1244.50 & 379.32 & 6 & 96.2 & 153.6 & 45.3 & 10.0 & 50.0 & 0.0603 \\
\hline A1249.00 & 380.70 & 6 & 92.7 & 184.1 & 26.6 & 10.0 & 50.0 & 0.0628 \\
\hline A1254.50 & 382.37 & 6 & 94.1 & 229.2 & 41.9 & 10.0 & 50.0 & 0.0622 \\
\hline A1260.45 & 384.19 & 5 & 98.3 & 159.3 & 43.3 & 10.0 & 50.0 & 0.1093 \\
\hline A1267.75 & 386.41 & 5 & 92.4 & 317.2 & -34.2 & 10.0 & 50.0 & 0.1187 \\
\hline A1270.65 & 387.29 & 5 & 97.5 & 178.3 & 43.1 & 10.0 & 50.0 & 0.0892 \\
\hline A 1275.20 & 388.68 & 5 & 97.6 & 222.3 & 30.7 & 10.0 & 50.0 & 0.0775 \\
\hline A1280.20 & 390.20 & 5 & 98.3 & 213.2 & 38.7 & 10.0 & 50.0 & 0.1118 \\
\hline A1285.45 & 391.81 & 5 & 97.4 & 268.8 & 62.0 & 10.0 & 50.0 & 0.0850 \\
\hline A1289.80 & 393.13 & 6 & 96.4 & 153.3 & 43.8 & 10.0 & 50.0 & 0.0584 \\
\hline A1296.65 & 395.22 & 4 & 98.0 & 252.0 & 41.7 & 20.0 & 50.0 & 0.0244 \\
\hline A 1300.40 & 396.36 & 6 & 95.0 & 20.5 & 64.1 & 10.0 & 50.0 & 0.0549 \\
\hline A1305.10 & 397.79 & 5 & 96.1 & 293.6 & 66.5 & 10.0 & 50.0 & 0.0320 \\
\hline A 1310.85 & 399.55 & 5 & 99.4 & 127.1 & -41.4 & 10.0 & 50.0 & 0.0812 \\
\hline A1316.50 & 401.27 & 5 & 96.9 & 233.8 & 50.9 & 10.0 & 50.0 & 0.0594 \\
\hline A1319.40 & 402.15 & 4 & 96.7 & 134.0 & 48.6 & 20.0 & 50.0 & 0.0279 \\
\hline A 1320.40 & 402.46 & 5 & 87.6 & 71.2 & 46.6 & 10.0 & 50.0 & 0.0472 \\
\hline A1324.60 & 403.74 & 5 & 97.3 & 219.9 & 54.6 & 10.0 & 50.0 & 0.0547 \\
\hline A1330.90 & 405.66 & 5 & 89.6 & 159.8 & 62.8 & 10.0 & 50.0 & 0.0310 \\
\hline A1337.35 & 407.62 & 5 & 99.2 & 67.7 & 45.2 & 10.0 & 50.0 & 0.0304 \\
\hline A1344.20 & 409.71 & 5 & 99.1 & 120.5 & 50.3 & 10.0 & 50.0 & 0.0270 \\
\hline A1349.10 & 411.21 & 5 & 98.9 & 168.4 & 55.4 & 10.0 & 50.0 & 0.0322 \\
\hline A 1350.10 & 411.51 & 5 & 97.8 & 124.2 & 84.0 & 10.0 & 50.0 & 0.0284 \\
\hline A1356.40 & 413.43 & 5 & 96.9 & 145.1 & 50.5 & 10.0 & 50.0 & 0.0198 \\
\hline A 1360.20 & 414.59 & 5 & 93.9 & 174.9 & 63.7 & 10.0 & 50.0 & 0.0136 \\
\hline A1365.50 & 416.20 & 5 & 96.3 & 266.6 & 9.6 & 10.0 & 50.0 & 0.0257 \\
\hline A1372.90 & 418.46 & 5 & 64.7 & 269.7 & 73.7 & 10.0 & 50.0 & 0.0151 \\
\hline A1378.80 & 420.26 & 3 & 82.9 & 155.8 & -17.6 & 30.0 & 50.0 & 0.0200 \\
\hline A1383.35 & 421.65 & 5 & 99.5 & 241.4 & -63.6 & 10.0 & 50.0 & 0.1402 \\
\hline A1387.40 & 422.88 & 5 & 96.1 & 22.8 & 58.7 & 10.0 & 50.0 & 0.0365 \\
\hline A1394.30 & 424.98 & 5 & 82.9 & 92.4 & 58.4 & 10.0 & 50.0 & 0.0247 \\
\hline A1400.30 & 426.81 & 5 & 95.5 & 171.9 & 64.1 & 10.0 & 50.0 & 0.0263 \\
\hline A1405.50 & 428.40 & 5 & 79.2 & 128.9 & 67.1 & 10.0 & 50.0 & 0.0198 \\
\hline A1410.70 & 429.98 & 5 & 97.6 & 282.7 & 52.1 & 10.0 & 50.0 & 0.0322 \\
\hline A1416.70 & 431.81 & 5 & 91.6 & 260.8 & 52.4 & 10.0 & 50.0 & 0.0312 \\
\hline A 1420.40 & 432.94 & 2 & 71.7 & 253.8 & -12.1 & 30.0 & 50.0 & 0.0089 \\
\hline A1424.60 & 434.22 & 1 & $?$ & 56.8 & -19.1 & 40.0 & 50.0 & 0.0069 \\
\hline A1430.60 & 436.05 & 1 & $?$ & 91.7 & -5.5 & 40.0 & 50.0 & 0.0302 \\
\hline A1436.90 & 437.97 & 5 & 98.7 & 117.0 & 50.3 & 10.0 & 50.0 & 0.0184 \\
\hline A 1440.44 & 439.05 & 5 & 96.9 & 136.7 & 66.4 & 10.0 & 50.0 & 0.0128 \\
\hline
\end{tabular}

Note: $\mathrm{N}=$ number of data used in each least-squares analysis; Var = percentage of the total variance in the selected data accounted for by the least-squares vector (dash indicates variance calculation not applicable); Dec, Inc $=$ declination and inclination of the magnetization vector; First, Last = first and last demagnetization step in millitesla; Jcomp $=$ intensity of least-squares magnetization.

er, because of the poor recovery rate down to $1050 \mathrm{ft}(320.0 \mathrm{~m})$ at Site AC, I could only sample the lowermost Kirkwood and uppermost Atlantic City Formations in limited locations. Although there are relatively strong and stable magnetizations present in the various sediment types within these sections, the wide distribution of samples makes it difficult to make any assessment in terms of magnetostratigraphy.

Below the disconformity at $1181 \mathrm{ft}(360.0 \mathrm{~m})$, silty clays of the Absecon Inlet Formation yield very consistent directions during progressive AF demagnetization (Fig. 4). None of the samples from this interval were rejected on the grounds of poor data quality. The intensity of magnetization ranges from 0.01 to $0.1 \mathrm{~mA} / \mathrm{m}$, and although this $130-\mathrm{ft}(39.6 \mathrm{~m})$ section is almost entirely normal in polarity, two reversed polarity magnetizations at 1267.75 and $1310.85 \mathrm{ft}(386.4$ and $399.5 \mathrm{~m}$ ) suggest that these sediments record an ancient magnetization.

From the disconformity at $1333 \mathrm{ft}(406.3 \mathrm{~m})$ to the bottom of the Site AC core (1452 ft [442.6 m]) in shelly clays of the middle Eocene Shark River Formation, the intensity of magnetization hardly varies and is on the order of $0.01 \mathrm{~mA} / \mathrm{m}$ (Table 3). Most of the 21 samples from this section yield straightforward demagnetization profiles (Fig. 4 ), although in a few samples secondary overprints were found. One part of this section, in particular from 1422 to $1431 \mathrm{ft}$ (433.4-436.2 $\mathrm{m}$ ), appears to contain a primary magnetization of reversed polarity that has been significantly contaminated by a steep normal downward overprint perhaps related to drilling (Fig. 4). In such samples, the net magnetization moves from the lower to upper hemisphere during progressive AF treatment from NRM to the limit of $50 \mathrm{mT}$. I interpret these data as indirect evidence for a reversed magnetization.

In summary, at Site AC, 59 whole-round samples have been progressively AF demagnetized and analyzed. Correlations to the GPTS were made based on the data from the 48 samples below the disconformity at $1181 \mathrm{ft}(360.0 \mathrm{~m})$ and the biostratigraphic data from Site AC. It is very difficult to assign a magnetochronology to the thick, predominantly normal polarity interval within the upper Eocene Absecon Inlet Formation (1200 ft [365.8 m] to disconformity at $1325 \mathrm{ft}$ [403.9 m], Fig. 4) and Shark River Formation below. In general, the sediments at a depth of $1181 \mathrm{ft}(360.0 \mathrm{~m})$ are no younger than Zone $\mathrm{P} 17$, as suggested by the planktonic foraminifer data (Miller et al., 1994a). Calcareous nannofossils indicate that Zone NP19/20 extends 


\section{ATLANTIC CITY}
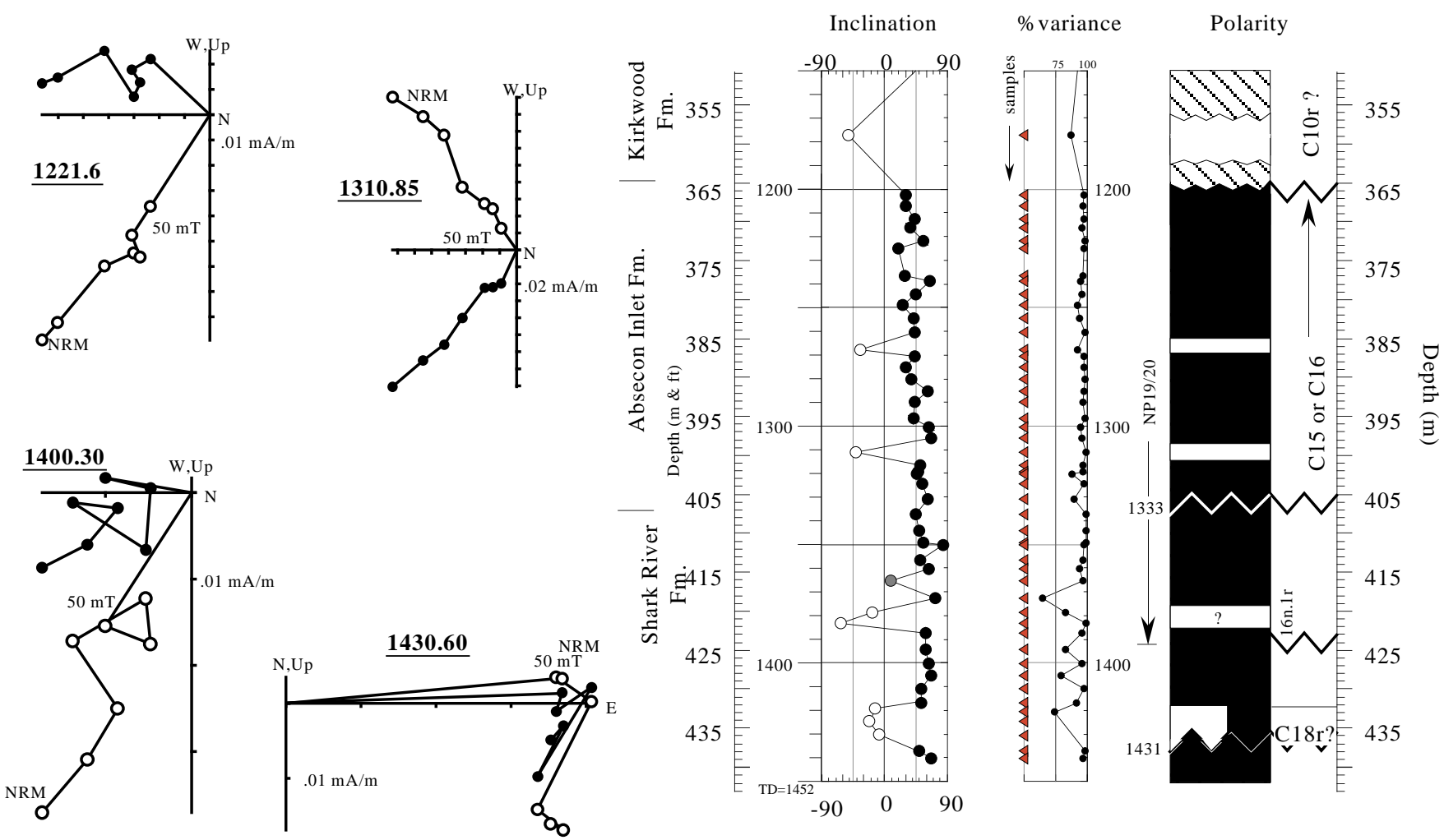

Figure 4. Progressive AF demagnetization and magnetostratigraphy of samples from Site AC (1150-1452 ft [350.5-442.6 m]). Demagnetization profiles in orthographic projection are shown at left (closed/open symbols $=$ horizontal/vertical projection, $\mathrm{mT}=$ millitesla). Inclination of remanence vs. depth shown on right along with percent variance (triangles give location of discrete samples). Polarity interpretation (black = normal, white $=$ reverse, hatch $=$ uninterpretable).

down to $1390 \mathrm{ft}$ (423.7 m; M.-P.Aubry, pers. comm., 1995), which, given the GPTS, means that the upper Eocene sediments at Site AC should be no older than Chron C16. Therefore, the best magnetostratigraphic age that can be assigned to the interval between 1181 and $1390 \mathrm{ft}$ (360.0 and $423.7 \mathrm{~m}$ ) is Chron C15n/r or C16n/r (Fig. 4). Such an assignment predicts much more reversed polarity magnetizations than is observed in these sediments. Whereas the thin reversed zone between 1375.85 and $1385.40 \mathrm{ft}(419.4$ and $422.3 \mathrm{~m})$ may represent C16n.1r (35.55-35.72 Ma; Table 4), the predominance of normal polarity magnetization at Site AC remains a mystery.

The lowest occurrence of Porticulasphaera semiinvoluta is at a depth of $1348 \mathrm{ft}(410.9 \mathrm{~m})$ and marks the base of planktonic foraminifer Zone P15 (Miller et al., 1994a). I therefore correlate the polarity reversals within the upper Shark River Formation (1325 and $1431 \mathrm{ft}$ [403.9 and 436.2 m)] to Chron C18n and C18r (Fig. 4). The thin reversed zone between 1375.85 and $1385.40 \mathrm{ft}$ (419.4 and $422.3 \mathrm{~m}$ ) may represent $\mathrm{C} 18 \mathrm{n} .1 \mathrm{r}(39.64-39.72 \mathrm{Ma}$; see Table 2). The field reversal at $1422.25 \mathrm{ft}(433.5 \mathrm{~m})$, which I infer from three overprinted but reversely magnetized samples, may represent the termination of Chron C18r (40.22 Ma).

\section{Cape May Site (Site CM)}

Site $\mathrm{CM}$ is located at $38^{\circ} 56^{\prime} \mathrm{N}, 74^{\circ} 53^{\prime} \mathrm{W}$ in the Coast Guard Training Center at a mean elevation of $1.5 \mathrm{~m}$ (Fig. 1). Drilling to $1500 \mathrm{ft}$ $(457.2 \mathrm{~m})$ recovered about $1129 \mathrm{ft}(344.1 \mathrm{~m})$ of core. Our study of this site is focused on the lower to middle Miocene Kirkwood Formation. Samples were taken from the more consolidated silty clay and sandy mud intervals below roughly $650 \mathrm{ft}(198.1 \mathrm{~m})$, which forms the aquifer confining units of this formation (Miller, et al., 1996). In addition, the unnamed upper and lower Oligocene glauconitic sands were sampled in two sections.

Silts and silty sands of lower Miocene Kirkwood Unit 2a (615$710 \mathrm{ft}$ [187.5-216.4 m]) are relatively strongly magnetized, but yield rather erratic demagnetization profiles. A consistent and stable magnetization in four samples was from in this unit (Fig. 5; Table 4), however these were too widely distributed stratigraphically to make any useful interpretation in terms of polarity history. The base of Kirkwood Unit $1 \mathrm{~b}$ was suitable for sampling between 905 and $927 \mathrm{ft}$ (275.8 and $282.5 \mathrm{~m})$. Here a strong $(0.1-1.0 \mathrm{~mA} / \mathrm{m})$ and stable magnetization is found that is normal in polarity throughout, with the exception of one reversed sample at $917.20 \mathrm{ft}(279.6 \mathrm{~m})$.

Between about 942 and $1062 \mathrm{ft}(287.1$ and $323.7 \mathrm{~m})$, the gray silty clays of Kirkwood Unit 1a sequence contain a magnetization that is relatively weak in intensity $(\sim 0.01 \mathrm{~mA} / \mathrm{m})$, but very consistent in direction during demagnetization. This unit features a thick normal polarity zone from 966 to $994 \mathrm{ft}(294.4-303.0 \mathrm{~m})$ in which magnetizations decay to the origin virtually unchanged in direction from the NRM (Fig. 5). It is conceivable that these sediments contain only a recent secondary magnetization. Continuing down through Unit 1a, the magnetizations remain weak and predominantly normal in polarity. However, near a lithological change to glauconitic clay $(\sim 1020 \mathrm{ft}$ [310.9 m]), there is a thin zone of reversed polarity (between 1018.0 and $1024.9 \mathrm{ft}$ [310.3 and $312.4 \mathrm{~m}$ ]; Table 4).

The Kirkwood equivalent, Kw0 sequence (1062-1180 ft [323.7$359.7 \mathrm{~m}]$ ), may be the only lower Miocene section at Cape May con- 
Table 3. Progressive demagnetization data used in constructing Site CM (Cape May) magnetostratigraphy.

\begin{tabular}{|c|c|c|c|c|c|c|c|c|}
\hline $\begin{array}{c}\text { Sample } \\
\text { name }\end{array}$ & $\begin{array}{l}\text { Depth } \\
\text { (m) }\end{array}$ & $\mathrm{N}$ & $\begin{array}{l}\text { Var } \\
(\%)\end{array}$ & $\begin{array}{l}\text { Dec } \\
\left({ }^{\circ}\right)\end{array}$ & $\begin{array}{l}\text { Inc } \\
\left({ }^{\circ}\right)\end{array}$ & $\begin{array}{l}\text { First } \\
(\mathrm{mT})\end{array}$ & $\begin{array}{l}\text { Last } \\
(\mathrm{mT})\end{array}$ & $\begin{array}{c}\text { Jcomp } \\
(\mathrm{mA} / \mathrm{m})\end{array}$ \\
\hline \multicolumn{9}{|l|}{ 150X-CM- } \\
\hline C625.50 & 190.65 & 6 & 87.5 & 141.3 & -46.6 & 20.0 & 70.0 & 0.6600 \\
\hline C649.60 & 198.00 & 4 & 87.1 & 209.9 & 31.0 & 20.0 & 50.0 & 0.3883 \\
\hline C669.75 & 204.14 & 6 & 88.8 & 342.9 & -21.2 & 20.0 & 70.0 & 0.7100 \\
\hline C693.65 & 211.42 & 6 & 97.6 & 130.0 & -56.3 & 20.0 & 70.0 & 0.0883 \\
\hline C906.40 & 276.27 & 6 & 91.4 & 154.1 & 29.2 & 10.0 & 70.0 & 0.8567 \\
\hline C916.40 & 279.32 & 7 & 96.2 & 137.0 & 59.2 & 15.0 & 90.0 & 2.8333 \\
\hline C917.20 & 279.56 & 6 & 75.2 & 26.3 & -22.0 & 30.0 & 90.0 & 0.1750 \\
\hline C926.50 & 282.40 & 6 & 98.8 & 202.2 & 73.7 & 25.0 & 90.0 & 2.8533 \\
\hline C966.10 & 294.47 & 5 & 98.8 & 172.0 & 43.5 & 20.0 & 50.0 & 0.3175 \\
\hline C971.30 & 296.05 & 5 & 96.1 & 278.3 & 43.8 & 20.0 & 50.0 & 0.0453 \\
\hline C974.10 & 296.91 & 5 & 95.4 & 306.5 & 63.9 & 20.0 & 50.0 & 0.0658 \\
\hline C976.10 & 297.52 & 5 & 95.9 & 183.4 & 18.9 & 20.0 & 50.0 & 0.0970 \\
\hline C980.30 & 298.80 & 5 & 87.3 & 307.2 & 53.9 & 20.0 & 50.0 & 0.0387 \\
\hline C982.90 & 299.59 & 5 & 93.0 & 1.7 & 63.9 & 20.0 & 50.0 & 0.0279 \\
\hline C984.60 & 300.11 & 6 & 97.8 & 279.3 & -5.8 & 20.0 & 50.0 & 0.0282 \\
\hline C987.10 & 300.87 & 6 & 96.1 & 150.9 & 61.1 & 20.0 & 50.0 & 0.0367 \\
\hline C988.50 & 301.29 & 5 & 99.2 & 295.2 & 53.8 & 20.0 & 50.0 & 0.0516 \\
\hline C994.60 & 303.15 & 5 & 92.0 & 14.7 & 27.4 & 20.0 & 50.0 & 0.0536 \\
\hline C1013.45 & 308.90 & 5 & 91.7 & 245.0 & 30.0 & 20.0 & 50.0 & 0.0169 \\
\hline C1014.90 & 309.34 & 5 & 97.7 & 238.1 & 28.9 & 20.0 & 50.0 & 0.0323 \\
\hline C1021.10 & 311.23 & 5 & 88.9 & 353.0 & -29.8 & 20.0 & 50.0 & 0.0243 \\
\hline C1024.25 & 312.19 & 5 & 86.3 & 107.8 & -23.7 & 20.0 & 50.0 & 0.0307 \\
\hline C1025.60 & 312.60 & 5 & 71.8 & 269.9 & 20.8 & 20.0 & 50.0 & 0.0263 \\
\hline C1030.80 & 314.19 & 5 & 85.4 & 279.8 & 26.4 & 20.0 & 50.0 & 0.0529 \\
\hline C1032.15 & 314.60 & 5 & 93.2 & 171.4 & 32.6 & 20.0 & 50.0 & 0.0300 \\
\hline C1037.75 & 316.31 & 5 & 90.0 & 155.0 & 27.3 & 20.0 & 50.0 & 0.0448 \\
\hline C1042.75 & 317.83 & 5 & 95.3 & 254.7 & 3.2 & 20.0 & 50.0 & 0.0967 \\
\hline C1046.50 & 318.97 & 5 & 86.4 & 185.7 & 5.3 & 20.0 & 50.0 & 0.0253 \\
\hline C1051.10 & 320.38 & 7 & 59.9 & 124.8 & 49.4 & 20.0 & 90.0 & 0.0983 \\
\hline C1060.50 & 323.24 & 8 & 98.4 & 41.1 & 19.3 & 20.0 & 90.0 & 0.5617 \\
\hline C1071.40 & 326.56 & 6 & 87.0 & 34.8 & -38.2 & 15.0 & 70.0 & 0.0101 \\
\hline C1092.90 & 333.12 & 8 & 95.9 & 219.6 & 62.3 & 20.0 & 90.0 & 0.6300 \\
\hline C1096.80 & 334.30 & 8 & 98.9 & 1.0 & -51.1 & 20.0 & 90.0 & 0.5233 \\
\hline C1114.35 & 339.65 & 7 & 98.6 & 352.1 & -57.9 & 20.0 & 90.0 & 2.9650 \\
\hline C1120.65 & 341.57 & 6 & 99.5 & 176.6 & 29.3 & 30.0 & 90.0 & 6.2583 \\
\hline C1121.30 & 341.77 & 6 & 99.8 & 147.8 & -35.2 & 20.0 & 50.0 & 150.2783 \\
\hline C1122.40 & 342.11 & 6 & 99.3 & 167.3 & -28.1 & 30.0 & 90.0 & 5.5167 \\
\hline C1126.10 & 343.24 & 5 & 99.2 & 188.9 & 69.1 & 30.0 & 90.0 & 6.7467 \\
\hline $\mathrm{C} 1140.10$ & 347.50 & 5 & 90.2 & 253.1 & 18.5 & 30.0 & 90.0 & 0.7150 \\
\hline C1144.10 & 348.72 & 6 & 85.0 & 131.3 & -5.7 & 15.0 & 70.0 & 0.2283 \\
\hline C1144.80 & 348.94 & 5 & 64.1 & 274.6 & 46.2 & 30.0 & 50.0 & 0.0873 \\
\hline C1154.20 & 351.80 & 7 & 73.5 & 29.7 & -26.3 & 25.0 & 85.0 & 0.2633 \\
\hline C1162.30 & 354.27 & 7 & 93.7 & 111.4 & -60.8 & 35.0 & 95.0 & 0.5783 \\
\hline $\mathrm{C} 1200.70$ & 365.97 & 4 & 100.0 & 141.9 & 85.5 & 10.0 & 48.0 & 14.0491 \\
\hline C1204.70 & 367.19 & 4 & 100.0 & 182.9 & 85.6 & 10.0 & 48.0 & 27.6805 \\
\hline C1208.70 & 368.41 & 5 & 100.0 & 233.5 & 84.7 & 10.0 & 48.0 & 15.7326 \\
\hline C1283.45 & 391.20 & 6 & 96.5 & 152.7 & -12.2 & 40.0 & 50.0 & 0.0133 \\
\hline C1306.50 & 398.22 & 7 & 99.3 & 163.0 & -27.8 & 20.0 & 50.0 & 0.1041 \\
\hline C1317.10 & 401.45 & 7 & 86.4 & 212.7 & 61.9 & 20.0 & 49.0 & 0.0595 \\
\hline C1323.00 & 403.25 & 7 & 94.0 & 256.3 & 39.1 & 20.0 & 49.0 & 0.0772 \\
\hline C1328.50 & 404.93 & 7 & 87.0 & 277.1 & 68.1 & 20.0 & 48.0 & 0.0381 \\
\hline $\mathrm{C} 1332.50$ & 406.15 & 6 & 95.5 & 134.0 & -56.6 & 20.0 & 48.0 & 0.0570 \\
\hline C1343.30 & 409.44 & 4 & 87.3 & 153.6 & -35.1 & 44.0 & 50.0 & 0.0232 \\
\hline C1436.60 & 437.88 & 5 & 98.2 & 275.7 & 47.0 & 20.0 & 50.0 & 0.0520 \\
\hline
\end{tabular}

Notes: $\mathrm{N}=$ number of data used in each least-squares analysis; Var = percentage of the total variance in the selected data accounted for by the least-squares vector (dash indicates variance calculation not applicable); Dec, Inc $=$ declination and inclination of the magnetization vector; First, Last $=$ first and last demagnetization step in millitesla; Jcomp $=$ intensity of least-squares magnetization.

taining what might be considered a continuous series of polarity reversals. Six of the 13 samples from this interval that yielded interpretable magnetizations are reversed in polarity. Glauconite sands at the top of this unit range in intensity from 1 to $100 \mathrm{~mA} / \mathrm{m}$ and yield very consistent demagnetization profiles. A reversed magnetization at $1121.30 \mathrm{ft}(341.7 \mathrm{~m})$ clearly demonstrates overprinting by a normal field (perhaps the present-day field), which is resolved between the NRM and applied field of $15 \mathrm{mT}$ (Fig. 5). Where the sands of this unit become more clay-rich below about $1140 \mathrm{ft}(347.5 \mathrm{~m})$, the magnetizations become less consistent during AF demagnetization. Two samples, at 1154.20 and $1162.30 \mathrm{ft}(351.8$ and $354.3 \mathrm{~m})$, suggest a zone of reversed polarity that closes out our lower Miocene sample set at Site CM.

There were a few isolated sections of upper Oligocene unnamed sediments at Site CM where paleomagnetic sampling was possible. Unfortunately, these green glauconitic sands between 1180 and 1270 $\mathrm{ft}$ (360 and $387.1 \mathrm{~m}$ ) yielded very inconsistent demagnetization profiles and are difficult to interpret. Three samples at the base of this unit contain a very strong magnetization pointed virtually straight down (Table 4) suggesting a drilling-related contamination. For the remainder of the upper Oligocene, quartz sands and glauconitic silty sands between 1220 and $1270 \mathrm{ft}$ (371.9 and $387.1 \mathrm{~m}$ ) yielded erratic magnetizations during analysis and could not be interpreted.

Magnetizations from the lower Oligocene (unnamed) at Site CM can be characterized as weak in intensity, but nonetheless consistent during AF treatment. Most yield normal polarity magnetizations (Table 4), but scattered through this section are samples that contain indirect evidence for reversed polarity magnetization. In these samples, for instance at $1283.45 \mathrm{ft}$ (391.2 m; Fig. 5), the direction of the net magnetization swings from the lower to upper hemisphere during progressive AF treatment, suggesting the existence of a reversed polarity magnetization with relatively high coercivity.

In summary, 97 whole-round and standard $6-\mathrm{cm}^{3}$ samples have been progressively demagnetized and analyzed at Site CM. There were many weak and/or spurious magnetizations at this site, leaving only 54 useful samples and many gaps in the overall stratigraphic coverage. However, the occurrence of reversed polarity magnetizations within the Kirkwood Formation and the unnamed Oligocene 
sediments suggest that secondary magnetizations have not totally contaminated the site, and therefore, the Cape May sediments have the potential to record an ancient magnetization. Using the planktonic foraminifer data and Sr-isotopes analyses at this site, there are two sections in which one can make reasonable correlations to the GPTS.

Within the Kw sequence of the Kirkwood Formation, there is a relatively thick section of normal polarity magnetizations from 966.10 to $1018.0 \mathrm{ft}$ (294.5-310.3 m; with sampling gap 995.0$1013.0 \mathrm{ft}[303.3-308.8 \mathrm{~m}])$. This is followed by a thin reversed polarity section between 1018.0 and $1024.9 \mathrm{ft}(310.3$ and $312.4 \mathrm{~m})$ followed by normal polarity magnetizations down to the disconformity at $1062 \mathrm{ft}(323.7 \mathrm{~m})$, marking the base of the Kirkwood sequence (Fig. 5). Sr-isotope dates from this interval average $20.7 \mathrm{Ma}$ (Sugarman et al., Chapter 12, this volume) and, together with the last occurrence of Globigerina angulisuturalis (Miller, et al., 1996; Liu et al., Chapter 10, this volume), suggest this polarity sequence may be correlative to Chrons C6n through C6An.1 (partim) (Table 2).

The polarity reversal sequence between 1062 and $1126 \mathrm{ft}(323.7$ and $343.2 \mathrm{~m}$ ) may correlate to Chrons 6An-6Ar. Given our age estimate of the overlying sediments ( $\mathrm{Kw} 1 \mathrm{a})$, and the planktonic foraminiferal data that suggest that lower Miocene Kirkwood Kw 0 is younger than Zone N4 (Liu et al., Chapter 10, this volume), the sediments in this unit cannot correlate to magnetozones older than Chron 6Ar. Between depths of 1094 and $1124 \mathrm{ft}$ (333.5 and $342.6 \mathrm{~m}$ ), Srisotope age dates range from 22.24 to $22.59 \mathrm{Ma}$ (Sugarman et al., Chapter 12 , this volume). These data suggest that this predominantly reversed polarity zone correlates to the older half of Chron C6Ar (Fig. 5). Given the age estimates of Kirkwood Units 1a and 0, it would appear that the hiatus represented by the disconformity at 1062 $\mathrm{ft}(323.7 \mathrm{~m})$ is on the order of $1 \mathrm{~m} . \mathrm{y}$.

\section{CONCLUSION}

At the three Leg 150X onshore sites, I have used a large-sample method to extract a record of paleomagnetic field polarity from the weakly magnetized sediments on the New Jersey coastal plain. From these data, field polarity reversals in lower Miocene, Oligocene, and Eocene sections were identified. Unfortunately, the many gaps in the physical and magnetostratigraphic coverage within the cores do not allow correlation between Sites IB, AC, and CM. However, at discrete stratigraphic levels within the cores, by incorporating biostratigraphic and Sr-isotope data, ages of sediments were determined based on a correlation to the GPTS.

At Site IB, a series of polarity intervals from Chron C20n through $\mathrm{C} 24 \mathrm{r}$ was found (C21r missing). Middle Eocene sediments at depths of 851.00 and $867.75 \mathrm{ft}(259.4$ and $264.5 \mathrm{~m})$ are dated as 46.28 and 49.60 Ma, respectively (C21n termination, C22n onset, ages referred to GPTS of Berggren et al., 1995). Two sequence boundaries at this site at 857 and $862 \mathrm{ft}(261.2$ and $262.7 \mathrm{~m})$ were found to be straddled by Chron $\mathrm{C} 22 \mathrm{n}$, suggesting the overall interruption of sedimentation at these levels was relatively brief $(<0.5$ m.y.). In the lower Eocene

\section{CAPE MAY}
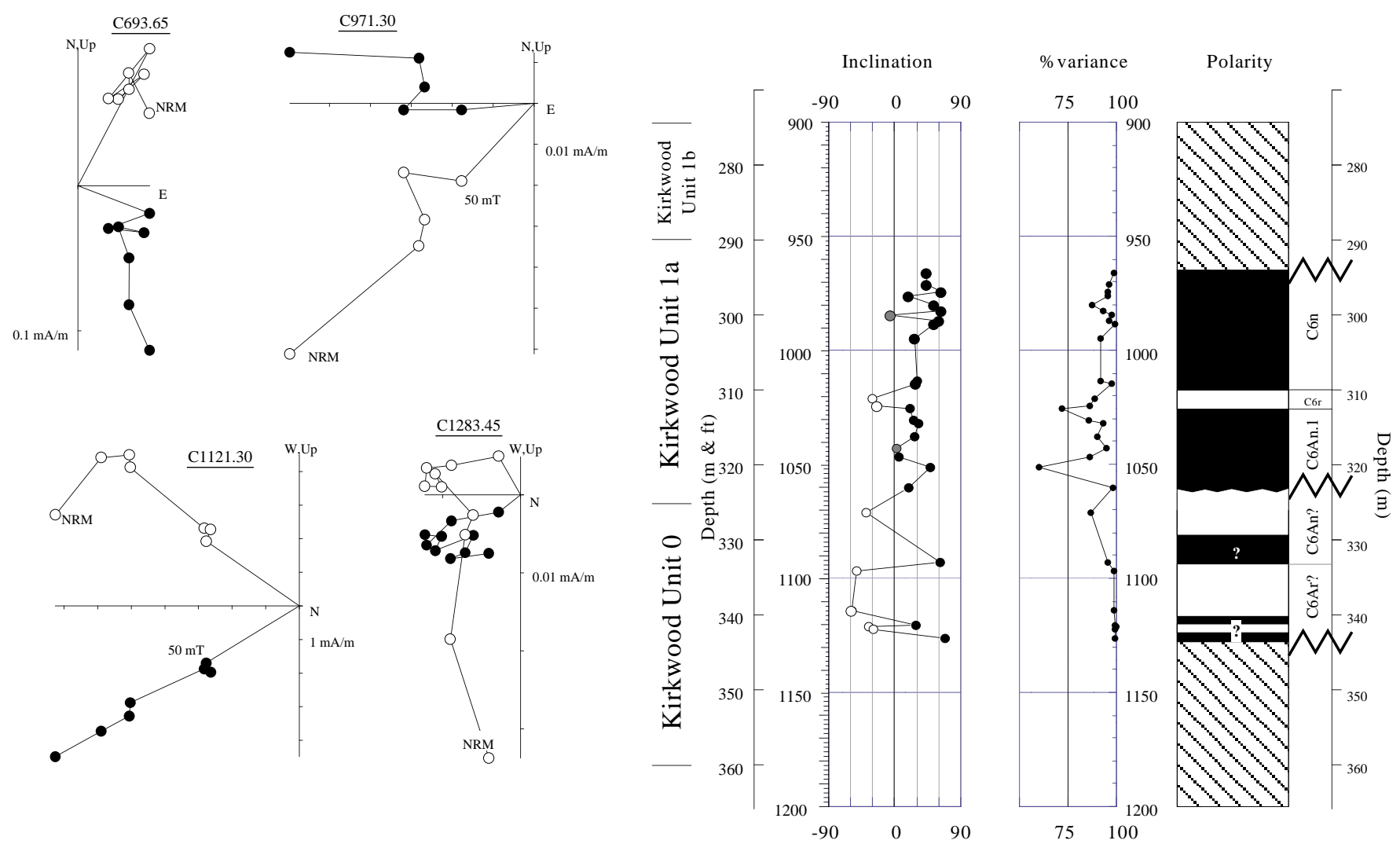

Figure 5. Progressive AF demagnetization and magnetostratigraphy of samples from Site CM (900-1200 ft [274.3-365.8 m]). Demagnetization profiles in orthographic projection are shown at left (closed/open symbols $=$ horizontal/vertical projection, $\mathrm{mT}=$ millitesla). Inclination of remanence vs. depth shown on right along with percent variance (triangles give location of discrete samples). Polarity interpretation (black $=$ normal, white $=$ reverse, hatch $=$ uninterpretable). 
Table 4. Polarity zonations and reversal boundary depths for Sites IB, $\mathrm{AC}$, and $\mathrm{CM}$.

\begin{tabular}{|c|c|c|c|c|}
\hline $\begin{array}{l}\text { Depth } \\
\text { (ft) }\end{array}$ & $\begin{array}{c}\text { Depth } \\
\text { (m) }\end{array}$ & Sense & Interpretation & $\begin{array}{l}\text { Age } \\
(\mathrm{Ma})\end{array}$ \\
\hline \multicolumn{5}{|c|}{ Site IB (Island Beach) } \\
\hline 851.00 & 259.4 & $N-R$ & $\mathrm{C} 2 \ln (\mathrm{T})$ & 46.28 \\
\hline 867.75 & 264.5 & $\mathrm{R}-\mathrm{N}$ & $\mathrm{C} 22 \mathrm{n}(\mathrm{O})$ & 49.60 \\
\hline 982.65 & 299.5 & $\mathrm{R}-\mathrm{N}$ & $\mathrm{C} 24 \mathrm{n} .1 \mathrm{r}(\mathrm{T})$ & 52.54 \\
\hline 984.00 & 299.9 & N-R & $\mathrm{C} 24 \mathrm{n} \cdot 2(\mathrm{O})$ & 52.79 \\
\hline \multicolumn{5}{|c|}{ Site AC (Atlantic City) } \\
\hline 1375.85 & 419.4 & $\mathrm{R}-\mathrm{N}$ & C16n.1r(T) & 35.55 \\
\hline 1385.40 & 422.3 & N-R & $\mathrm{C} 16 \mathrm{n} .1 \mathrm{r}(\mathrm{O})$ & 35.72 \\
\hline \multicolumn{5}{|c|}{ Site CM (Cape May) } \\
\hline 1018.00 & 310.3 & $\mathrm{R}-\mathrm{N}$ & $\mathrm{C} 6 \mathrm{n}(\mathrm{O})$ & 20.45 \\
\hline 1024.90 & 312.4 & N-R & C6An.1(T) & 20.88 \\
\hline
\end{tabular}

Notes: Ages from Berggren et al. (1995); chronostratigraphic notation from Cande and Kent (1992). $\mathrm{O}=$ onset, $\mathrm{T}=$ termination, $\mathrm{N}=$ normal, and $\mathrm{R}=$ reverse

section, sediments at 982.65 and $984.00 \mathrm{ft}$ (299.5 and $299.9 \mathrm{~m})$ are dated as 52.54 and $52.79 \mathrm{Ma}$, respectively. Middle Eocene sediments between 1375.85 and $1385.40 \mathrm{ft}(419.4$ and $422.3 \mathrm{~m})$ at Site $\mathrm{AC}$ are correlated to Chron C16n.1r (35.55-35.72 Ma). A portion of the lower Miocene Kirkwood Formation at Site CM contained a record of Chrons C6n through C6Ar. Sediments at depths of 1018.00 and $1024.90 \mathrm{ft}$ (310.3 and $312.4 \mathrm{~m}$ ) are dated as 20.45 and $20.88 \mathrm{Ma}$, respectively.

\section{ACKNOWLEDGMENTS}

I am greatly indebted to Dr. Dennis Kent for his advice and the use of the paleomagnetics lab at LDEO throughout this study. I would also like to thank Don Queen, Gene Cobb Sr., and the rest of the USGS drilling crew for their hard work. Special thanks go to Dr. Greg Mountain, Dr. Jim Browning, Steve Pekar, Dr. Marie-Pierre Aubry, and Dr. Pete Sugarman for their advice and supporting data. Drs. Jeff Gee and Don McNeil made very thoughtful reviews of the manuscript. Gilberto Mello made most of the paleomagnetic measurements at the LDEO laboratory. Finally, I would like to thank Dr. Ken Miller for the years of advice, financial support, and for overseeing the New Jersey Transect project. This study was supported by JOI/USSAC.

\section{REFERENCES}

Berggren, W.A., Kent, D.V., Swisher, C.C., III, and Aubry, M.-P., 1995. A revised Cenozoic geochronology and chronostratigraphy. In Berggren, W.A., Kent, D.V., Aubry, M.-P., and Hardenbol, J. (Eds.), Geochronology, Time Scales and Global Stratigraphic Correlation. Spec. Publ.Soc. Econ. Paleontol. Mineral., 54:129-212.

Cande, S.C. and Kent, D.V., 1992. A new geomagnetic polarity time scale for the Late Cretaceous and Cenozoic. J. Geophys. Res., 97:13,917-13,951.

Ellwood, B.B., McPherson, J.G., Sen Gupta, B.K., and Matthews, M., 1986. The proposed Eocene-Oligocene stratotype, S.W. Alabama: not ideal due to magnetostratigraphic inconsistencies. Palaios, 1:417-419.

Kirschvink, J.L., 1980. The least-squares line and plane and the analysis of paleomagnetic data. Geophys. J.R. Astron. Soc., 62:699-718.

Miller, K.G., et al., 1996. Proc. ODP, Init. Repts., 150X (Suppl.): College Station, TX (Ocean Drilling Program)

Miller, K.G., Browning, J.V., Liu, C., Sugarman, P., Kent, D.V., Van Fossen, M., Queen, D., Goss, M., Gwynn, D., Mullikin, L., Feigenson, M.D., Aubry, M.-P., and Burckle, L.D., 1994a. Atlantic City site report. In Miller, K.G., et al., Proc. ODP, Init. Repts., 150X: College Station, TX (Ocean Drilling Program), 35-55.

Miller, K.G., Kent, D.V., Brower, A.N., Bybell, L.M., Feigenson, M.D., Olsson, R.K., and Poore, R.Z., 1990. Eocene-Oligocene sea-level changes on the New Jersey coastal plain linked to the deep-sea record. Geol. Soc. Am. Bull., 102:331-339.

Miller, K.G. and Mountain, G.S., 1994. Global sea-level change and the New Jersey margin. In Mountain, G.S., Miller, K.G., Blum, P., et al., Proc. ODP, Init. Repts., 150: College Station, TX (Ocean Drilling Program), $11-20$.

Miller, K.G., Sugarman, P., Van Fossen, M., Liu, C., Browning, J.V., Queen, D., Aubry, M.-P., Burckle, L.D., Goss, M., and Bukry, D., 1994b. Island Beach site report. In Miller, K.G., et al., Proc. ODP, Init. Repts., 150X: College Station, TX (Ocean Drilling Program), 5-33.

Miller, K. G., Thompson, P.R., and Kent, D.V., 1993. Integrated late EoceneOligocene stratigraphy of the Alabama coastal plain: correlation of hiatuses and stratal surfaces to glacioeustatic lowerings. Paleoceanography, 8:313-331.

Van Fossen, M.C. and Urbat, M., 1996. Magnetostratigraphy of Miocene and Pleistocene sediments on the New Jersey slope. In Mountain, G.S., Miller, K.G., Blum, P., Poag, C.W., and Twichell, D.C. (Eds.), Proc. ODP, Sci. Results, 150: College Station, TX (Ocean Drilling Program), 129143.

Date of initial receipt: 29 January 1996

Date of acceptance: 15 October 1996

Ms 150XSR-318 